Short Review: Open Access

\title{
The Neuroimaging Findings of Internet Gaming Disorder
}

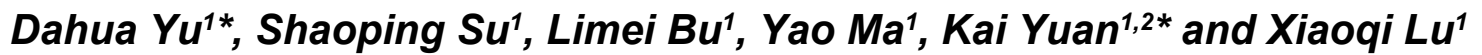 \\ ${ }^{1}$ Inner Mongolia Key Laboratory of Pattern Recognition and Intelligent Image Processing, Inner Mongolia University \\ of Science and Technology, China \\ ${ }^{2}$ Life Sciences Research Center, School of Life Science and Technology, Xidian University, China
}

*Corresponding author: Dahua Yu, Inner Mongolia Key Laboratory of Pattern Recognition and Intelligent Image Processing, School of Information Engineering, Inner Mongolia University of Science and Technology, Baotou, Inner Mongolia 014010, China, Tel: +86 472 5951563, Fax: +86 472 5951563, E-mail: fmydh@imust.edu.cn

Kai Yuan, Life Sciences Research Center, School of Life Science and Technology, Xidian University, Xi'an, Shaanxi 710071, Inner Mongolia Key Laboratory of Pattern Recognition and Intelligent Image Processing, School of Information Engineering, Inner Mongolia University of Science and Technology, Baotou, China, Tel: +86 29 81891070, Fax: +86 29 81891060,E-mail:kyuan@xidian.edu.cn

\begin{abstract}
Internet gaming disorder (IGD) was confirmed as a condition warranting more clinical research in the fifth edition of the Diagnostic and Statistical Manual of Mental Disorders (DSM-V) Section III, which was a great progress for IGD studies. In China, there are about 24 million adolescents with IGD. IGD is involved in academic failure, impairment of work performance and psychological comorbidity including anger problems, depression and anxiety disorders. With the help of advanced neuroimaging technology, the neuroimaging findings of IGD revealed structural and functional changes in the brain, which was similar as other substance related and behavioral addiction. The reward, craving, decision-making and memory circuits in the brain were investigated to explain the neural mechanism of IGD. In this paper, we reviewed several neuroimaging studies in IGD, which may improve the understanding of pathophysiology in IGD.
\end{abstract}

\section{Keywords}

Internet gaming disorder (IGD), MRI, Positron emission tomography (PET), Electroencephalography (EEG)

\section{Introduction}

Internet gaming provided a kind of popular leisure activities to make people relaxed and improve their quality of life, which may even enhance cognitive control in older adults [1]. However, excessive play of Internet games may lead to negative consequences, such as academic failure, impairment of work performance and psychological comorbidity including anger problems, depression and anxiety disorders [2]. The $36^{\text {th }}$ Statistical report on internet development in China released by China Internet Network Information Center (CNNIC) reported there were 668 million Internet users in China, in which Internet users aged 20-29 years were the largest proportion as $31.4 \%$ [3]. The incidence of IGD is about $14.1 \%$, which means there were over 24 million adolescents with IGD in China. Internet gaming disorder (IGD) was confirmed as a condition warranting more clinical research in the fifth edition of the Diagnostic and Statistical
Manual of Mental Disorders (DSM-V) Section III, which was a great progress for IGD studies [4].

IGD may impact normal life activities and be related to neglect family and social responsibilities in the individuals with IGD. The understanding of neurological mechanism of IGD remains unclear, which may cause the treatment of IGD moves slowly. In several institutions of China, extreme treatment of IGD led to the tragedy of teenagers injury and even death, which was reported in the journal Science [5]. With the help of advanced neuroimaging technology, previous neuroimaging findings of IGD revealed structural and functional changes in the brain, which was similar as other substance related and behavioral addiction [6,7]. The reward, craving, decisionmaking and memory circuits in the brain were investigated to explain the neural mechanism of IGD. IGD was a kind of specific behavioral addiction disorder, which was hard to build animal model. Previous studies confirmed both substance and behaviors may cause structural and functional changes in the reward circuit of brain [8]. Substance and natural rewards may affect similar brain circuits and regions. The similar changed pattern of cognitive control and behavior were found in the individuals with IGD and other substance related addiction and gambling disorder. In DSM-5, gambling disorder is the only non-substance-related addiction disorder which may help us to understand the concept of behavioral addiction. League of Legends (LOL), World of Warcraft (WOW) and Cross Fire (CF) etc. are popular Internet games in China, which are multiplayers games involving competition, cooperation social interactions [9]. The individuals with IGD typically spend more than 8 hours per day to play Internet games and neglect of other activities, such as school, work, family obligations, even food or sleep. IGD was referred to as Internet use disorder, problematic Internet use, Internet addiction disorder (IAD), or gaming addiction, which attracted research interests across the whole world and lacked clear diagnostic criteria. It is a great progress of IGD study that IGD was identified in DSM-5 as a condition warranting more clinical research [10,11]. Proposed diagnostic criteria of IGD in DSM-5 focused on Internet gaming excluding gambling Internet games, required activities in a business 
or profession by using the Internet, sexual Internet sites visiting or other recreational or social Internet use. The debate on the diagnostic criteria definition of IGD is still carrying on. The significance of imaging data studies may provide a potential diagnostic means for IGD. Previous studies revealed IGD may share similar neurological mechanisms with substance related addiction, which were mainly related to the reward circuit, cognitive control circuit, decision making circuit and memory circuit in the brain [12-15]. As a behavior related addiction disorder, IGD may be related to more complex neural mechanisms and interact with brain maturation during adolescence. In this paper, we reviewed several neuroimaging studies in IGD, which may improve the understanding of pathophysiology in IGD and help to modify the diagnostic criteria of IGD.

\section{PET findings}

Positron emission tomography (PET) is a powerful tool to image functional brain activity in previous addiction researches, especially substance related cue induced craving with dopamine increase in the striatum $[6,12]$. In PET studies of IGD, dopamine released in the striatum was also confirmed during playing a video game by using ${ }^{11} \mathrm{C}$-labelled raclopride [13]. Game related cue may induce increased activity in the striatum and craving score $[16,17]$. The regional cerebral glucose metabolism during resting state in the young individuals with IAD were investigated by using ${ }^{18} \mathrm{~F}$-fluorodeoxyglucose [18]. Compared with health normal control, young individuals with IAD showed increased glucose metabolism in the right middle orbitofrontal gyrus, left caudate nucleus, and right insula, and decreased glucose metabolism in the bilateral postcentral gyrus, left precentrai gyrus, and bilateral occipital regions [18]. Moreover, the level of striatal dopamine D2 receptors in individuals with IGD was assessed by using ${ }^{11} \mathrm{C}$-labelled raclopride. IGD individuals showed reduced dopamine D2 receptor availability in the bilateral dorsal caudate and right putamen as subdivisions of the striatum, which were negative correlated with the severity of IGD [19]. The changes of D2 receptor availability might prove behaviors as IGD can cause the changes in the reward circuit of brain, which may improve the clinical diagnosis and treatment of IGD [8]. A single photon emission computed tomography (SPECT) study measure striatal dopamine transporter (DAT) levels in individuals IGD by using ${ }^{99 \mathrm{~m}} \mathrm{Tc}$ TRODAT-1 [20]. The ${ }^{99 \mathrm{~m} T c-T R O D A T-1}$ uptake ratio of striatum and DAT levels of the striatum was greatly reduced in the individuals with IGD [20]. Taken together, PET studies of IGD revealed functional changes in the dopamine systems and shared similar neurobiological mechanism with substance related addiction [6].

\section{Structural findings by MRI}

By voxel-based morphometry (VBM), IGD group showed lower density in the left anterior cingulate cortex (ACC), left posterior cingulate cortex, left insula, and left lingual gyrus [21]. Yuan, et al. revealed decreased gray matter volumes in the bilateral dorsolateral prefrontal cortex (DLPFC), the supplementary motor area (SMA), the orbitofrontal cortex (OFC), the cerebellum and the left rostral ACC (rACC) in IGD group [22]. Decreased gray matter volumes in the right OFC, bilateral insula, ACC, and SMA of individuals with IGD were confirmed [23,24]. The gray matter volume of ACC was negative correlated with the incongruent response errors of Stroop task in IGD group [24]. Decreased gray matter volume in similar brain regions were revealed by several other VBM studies of IGD [25-27]. Moreover, compared with health normal controls, the individuals with IGD showed increased cortical thickness in the left precentral cortex, precuneus, middle frontal cortex, inferior temporal and middle temporal cortices and decreased cortical thicknesses of the left lateral OFC, insula, lingual gyrus, the right postcentral gyrus, entorhinal cortex and inferior parietal cortex [28]. In correlation analysis, the cortical thicknesses of the left precentral cortex, precuneus and lingual gyrus were correlated with duration of IGD and the cortical thickness of the OFC was correlated with the impaired task performance during the color-word Stroop task [28].

The structural changes in the white matter (WM) maybe described by multiple DTI-derived indices (such as fractional anisotropy (FA), mean diffusivity (MD), radial diffusivity (RD) and axial diffusivity $(\mathrm{AD})$ ) analysis, that may offer an unique insight to measure specific alterations in the WM $[29,30]$. Yuan, et al. revealed increased FA values of the left posterior limb of the internal capsule (PLIC) and decreased FA value in the right parahippocampal gyrus (PHG) in IAD subjects compared with healthy controls [22]. Correlation analysis showed the increased FA values in the left PLIC were correlated with the duration of IGD [22]. Reduced FA values in the right genu of corpus callosum (CC), bilateral frontal lobe white matter, and right external capsule in individuals with IGD were found by Weng, et al. [23]. Lin, et al. found reduced FA values in IGD individuals may be caused by an increase in $\mathrm{RD}$ rather than in $\mathrm{AD}$ [31]. In addition, Xing, et al. investigate the correlation between cognitive control and brain regional changes by using fiber tracking and color-word Stroop task to abnormalities [32]. IGD individuals showed decreased FA values in the right salience network (SN), which were negatively correlated with the errors during the incongruent condition in color-word Stroop task [32].

\section{Functional findings by MRI}

Functional MRI (fMRI) measures blood-oxygen-level dependent (BOLD) to investigate activity in brain regions during the resting state and task conditions, which provide a noninvasive way to measure the neural responses [33]. Especially, fMRI studies during the resting state may serve as a neural biomarker to reflect the progress of disease and help to access the neural responses in task conditions [34]. Local changes of functional characteristic during the resting state in IGD group were investigated by amplitude of low frequency fluctuation (ALFF) method and Regional homogeneity (ReHo) methods. Yuan, et al. found IDG group showed significant increased ALFF values in the left medial orbitofrontal cortex (OFC), the left precuneus, the left supplementary motor area (SMA), the right parahippocampal gyrus (PHG) and the bilateral middle cingulate cortex (MCC) [35]. The ALFF values of the left medial OFC, left precuneus were correlated with the duration of IGD, and the ALFF values of the left medial OFC was correlated with colorword Stroop test performance [35]. Decreased ReHo in the right superior temporal gyrus, temporal, occipital and parietal brain regions and increased ReHo in the brainstem, inferior parietal lobule, cerebellum, middle frontal gyrus, posterior cingulate cortex (PCC) were reported in IGD group compared with control group [36-38]. Furthermore, inter-regional connections between brain regions were investigated by functional connectivity (FC) and Granger causal analysis (GCA) in IGD. FC, GCA and fiber tracking methods were combined to identify the changes of core brain network during the resting state in IGD [39]. Compared with control group, the changes of functional connectivity within central executive networks and effective connectivity within salience network were found in IGD group, compared with the changes of structural connectivity within salience network and right central executive network [39]. In addition, previous IGD studies reported the changes in the default mode network [40], executive control network [41], functional connectivity of the amygdala [42] and fronto-striatal network [25] during the resting state. Voxel-mirrored homotopic connectivity (VMHC) analysis revealed decreased VMHC in the DLPFC, which was negatively correlated with the duration of IGD [43].

Task-related fMRI study may access BOLD signals in response to specific events, which provides direct evidence to reflect cognitive conditions about diseases. For IGD studies, cue-induced craving paradigm with visual picture or video was classic experimental design, which may help to investigate the changes in the reward circuit, inhibitory control circuit, decision making circuit and memory circuit etc. $[16,17,44]$. Changed brain activation in the right orbitofrontal cortex, right nucleus accumbens, bilateral anterior cingulate and medial frontal cortex, right dorsolateral prefrontal cortex, and right caudate nucleus were reported in IGD group when they watched gaming pictures and mosaic pictures as stimuli to evaluate the cue-induced gaming urge [16]. The brain activations were 
positively correlated with self-reported gaming urge and recalling of gaming experience provoked by the WOW pictures [16]. Dong, et al. found IGD individuals showed increased activation in OFC in monetary gain trials and decreased anterior cingulate activation in monetary loss trials compared with health controls, which may be involved in changed reward system sensitivity in IGD [45]. Impaired effective connectivity within the response inhibition network as a part of frontal-basal ganglia pathway was confirmed in IGD subjects by using dynamic causal modeling (DCM) [46]. Dong, et al. used Stroop task to reveal increased 'Stroop effect'-related activity in the anterior and posterior cingulate cortices of IGD subjects, which may reflect decreased response inhibition efficiency [47].

\section{Conclusions}

In summary, structural findings revealed changed structural integrity, while functional findings revealed impaired functional integrity and efficiency of IGD relevant brain networks in both intra-regional and inter-regional level, which were similar to substance related addiction $[6,7]$. The structural and functional findings of IGD may suggest IGD might share similar neurobiological mechanism with substance related addiction. Adolescence from 12 to 20 years old were proved as the most likely to occur IGD [4]. The central nervous system of adolescent is undergoing a series of significant neurobiological changes of brain maturation, which makes the brains in the period from adolescence to adulthood relatively labile [48]. The structural and functional characteristic of nervous system in adolescent was especially easy to be influenced by outside influences, such as IGD [14,15,49]. Previous neuroimaging findings improved our understanding of IGD. However, more multi-model neuroimaging studies are needed to reveal the neural mechanism of IGD.

\section{Acknowledgments}

This paper is supported by the National Natural Science Foundation of China under Grant nos. 81571751, 81571753, 61502376, 81401478, 81401488, 81470816, 81471737, 81301281, 81271546, 81271549, the Natural Science Basic Research Plan in Shaanxi Province of China under Grant no. 2014JQ4118, and the Fundamental Research Funds for the Central Universities under the Grant nos. JB151204, JB121405, the Natural Science Foundation of Inner Mongolia under Grant no. 2014BS0610, the Innovation Fund Project of Inner Mongolia University of Science and Technology Nos. 2015QNGG03, 2014QDL002, General Financial Grant the China Post- doctoral Science Foundation under Grant no. 2014M552416.

\section{References}

1. Anguera JA, Boccanfuso J, Rintoul JL, Al-Hashimi O, Faraji F, et al. (2013) Video game training enhances cognitive control in older adults. Nature 501 97-101.

2. D Griffiths M, J Kuss D, L King D (2012) Video game addiction: Past, present and future. Current Psychiatry Reviews 8: 308-318.

3. CNNIC (2015) 36th Statistical report on internet development in China. China Internet Network Information Center (CNNIC), China.

4. Association DAP (2013) Diagnostic and statistical manual of mental disorders. Arlington: American Psychiatric Publishing

5. Stone $R$ (2009) Science in society. China reins in wilder impulses in treatment of 'Internet addiction'. Science 324: 1630-1631.

6. Volkow ND, Morales M (2015) The Brain on Drugs: From Reward to Addiction Cell 162: 712-725

7. Volkow ND, Wang GJ, Fowler JS, Tomasi D, Telang F (2011) Addiction: beyond dopamine reward circuitry. Proc Natl Acad Sci USA 108: 1503715042.

8. Holden C (2001) 'Behavioral' addictions: do they exist? Science 294: 980982

9. CNNIC (2014) Internet behavior study in Chinese adolescent. China Internet Network Information Center (CNNIC), China.

10. Petry NM, O'Brien CP (2013) Internet gaming disorder and the DSM-5. Addiction 108: 1186-1187.

11. Block JJ (2008) Issues for DSM-V: internet addiction. Am J Psychiatry 165: 306-307.
12. Volkow N, Tomasi D, Wang G, Logan J, Alexoff D, et al. (2014) Stimulantinduced dopamine increases are markedly blunted in active cocaine abusers. Mol Psychiatry 19: 1037-1043.

13. Koepp MJ, Gunn RN, Lawrence AD, Cunningham VJ, Dagher A, et al. (1998) Evidence for striatal dopamine release during a video game. Nature 393: 266268.

14. Lydon DM, Wilson SJ, Child A, Geier CF (2014) Adolescent brain maturation and smoking: what we know and where we're headed. Neurosci Biobehav Rev 45: 323-342.

15. Crews F, He J, Hodge C (2007) Adolescent cortical development: a critical period of vulnerability for addiction. Pharmacol Biochem Behav 86: 189-199.

16. Ko CH, Liu GC, Hsiao S, Yen JY, Yang MJ, et al. (2009) Brain activities associated with gaming urge of online gaming addiction. J Psychiatr Res 43: 739-747.

17. Ko CH, Liu GC, Yen JY, Chen CY, Yen CF, et al. (2013) Brain correlates of craving for online gaming under cue exposure in subjects with Internet gaming addiction and in remitted subjects. Addict Biol 18: 559-569.

18. Park HS, Kim SH, Bang SA, Yoon EJ, Cho SS, et al. (2010) Altered Regional Cerebral Glucose Metabolism in Internet Game Overusers: A18ffluorodeoxyglucose Positron Emission Tomography Study. CNS Spectrum 15: 159-166.

19. Kim SH, Baik SH, Park CS, Kim SJ, Choi SW, et al. (2011) Reduced striatal dopamine D2 receptors in people with Internet addiction. Neuroreport 22: 407-411.

20. Hou H, Jia S, Hu S, Fan R, Sun W, et al. (2012) Reduced striatal dopamine transporters in people with internet addiction disorder. J Biomed Biotechnol 2012: 854524 .

21. Zhou Y, Lin FC, Du YS, Qin LD, Zhao ZM, et al. (2011) Gray matter abnormalities in Internet addiction: a voxel-based morphometry study. Eur J Radiol 79: 92-95.

22. Yuan K, Qin W, Wang G, Zeng F, Zhao L, et al. (2011) Microstructure abnormalities in adolescents with internet addiction disorder. PLoS One 6 : e20708

23. Weng CB, Qian RB, Fu XM, Lin B, Han XP, et al. (2013) Gray matter and white matter abnormalities in online game addiction. Eur $\mathrm{J}$ Radiol 82: 13081312.

24. Wang H, Jin C, Yuan K, Shakir TM, Mao C, et al. (2015) The alteration of gray matter volume and cognitive control in adolescents with internet gaming disorder. Front Behav Neurosci 9: 64

25. Kühn S, Gallinat J (2015) Brains online: structural and functional correlates of habitual Internet use. Addict Biol 20: 415-422.

26. Jin C, Zhang T, Cai C, Bi Y, Li Y, et al. (2015) Abnormal prefrontal cortex resting state functional connectivity and severity of internet gaming disorder. Brain Imaging Bbehav.

27. Altbäcker A, Plózer E, Darnai G, Perlaki G, et al. (2015) Problematic internet use is associated with structural alterations in the brain reward system in females. Brain Imaging Behav.

28. Yuan K, Cheng P, Dong T, Bi Y, Xing L, et al. (2013) Cortical thickness abnormalities in late adolescence with online gaming addiction. PLoS One 8: e53055.

29. Thomason ME, Thompson PM (2011) Diffusion imaging, white matter, and psychopathology. Annu Rev Clin Psychol 7: 63-85.

30. Alexander AL, Lee JE, Lazar M, Field AS (2007) Diffusion tensor imaging of the brain. Neurotherapeutics 4: 316-329.

31. Lin F, Zhou Y, Du Y, Qin L, Zhao Z, et al. (2012) Abnormal White Matter Integrity in Adolescents with Internet Addiction Disorder: A Tract-Based Spatial Statistics Study. PloS one 7: e30253.

32. Xing L, Yuan K, Bi Y, Yin J, Cai C, et al. (2014) Reduced fiber integrity and cognitive control in adolescents with internet gaming disorder. Brain Res 1586: 109-117.

33. Fox MD, Raichle ME (2007) Spontaneous fluctuations in brain activity observed with functional magnetic resonance imaging. Nat Rev Neurosci 8: 700-711.

34. Wu QZ, Li DM, Kuang WH, Zhang TJ, Lui S, et al. (2011) Abnormal regional spontaneous neural activity in treatment-refractory depression revealed by resting-state fMRI. Hum Brain Mapp 32: 1290-1299.

35. Yuan K, Jin C, Cheng P, Yang X, Dong T, et al. (2013) Amplitude of low frequency fluctuation abnormalities in adolescents with online gaming addiction. PLoS One 8: e78708.

36. Liu J, Gao X, Osunde I, Li X, Zhou S, et al. (2010) Increased regional homogeneity in internet addiction disorder a resting state functional magnetic resonance imaging study (2009). Chin Med J (Engl) 123: 1904-1908.

37. Dong G, Huang J, Du X (2012) Alterations in regional homogeneity of restingstate brain activity in internet gaming addicts. Behav Brain Funct 8: 41. 
38. Kim H, Kim YK, Gwak AR, Lim JA, Lee JY, et al. (2015) Resting-state regiona homogeneity as a biological marker for patients with Internet gaming disorder: a comparison with patients with alcohol use disorder and healthy controls. Prog NeuroPsychopharmacol Biol Psychiatry 60: 104-111.

39. Yuan K, Qin W, Yu D, Bi Y, Xing L, et al. (2016) Core brain networks interactions and cognitive control in internet gaming disorder individuals in late adolescence/early adulthood. Brain Struct Funct 221:1427-1442.

40. Ding Wn, Sun Jh, Sun Yw, Zhou Y, Li L, et al. (2013) Altered default network resting-state functional connectivity in adolescents with Internet gaming addiction. PloS one 8: e59902.

41. Dong G, Lin X, Potenza MN (2015) Decreased functional connectivity in an executive control network is related to impaired executive function in Internet gaming disorder. Prog NeuroPsychopharmacol Biol Psychiatry 57: 76-85.

42. Ko CH, Hsieh TJ, Wang PW, Lin WC, Yen CF, et al. (2015) Altered gray matter density and disrupted functional connectivity of the amygdala in adults with Internet gaming disorder. Prog NeuroPsychopharmacol Biol Psychiatry 57:185-192.
43. Bi Y, Yuan K, Feng D, Xing L, Li Y, et al. (2015) Disrupted inter-hemispheric functional and structural coupling in Internet addiction adolescents. Psychiatry Res 234: 157-163.

44. Yuan K, Qin W, Liu Y, Tian J (2011) Internet addiction: Neuroimaging findings. Commun Integr Biol 4: 637-639.

45. Dong G, Huang J, Du X (2011) Enhanced reward sensitivity and decreased loss sensitivity in Internet addicts: an fMRI study during a guessing task. J Psychiatr Res 45: 1525-1529.

46. Li B, Friston KJ, Liu J, Liu Y, Zhang G, et al. (2014) Impaired frontal-basal ganglia connectivity in adolescents with internet addiction. Sci Rep 4: 5027.

47. Dong G, DeVito E, Du X, Cui Z (2012) Impaired inhibitory control in "internet addiction disorder": A functional magnetic resonance imaging study. Psychiatry Res 203: 153-158.

48. Paus T (2005) Mapping brain maturation and cognitive development during adolescence. Trends Cogn Sci 9: 60-68.

49. Gulley JM, Juraska JM (2013) The effects of abused drugs on adolescent development of corticolimbic circuitry and behavior. Neuroscience 249: 3-20. 